\title{
Adoption of Additive Manufacturing Approaches: The Case of Manufacturing SMEs
}

\author{
Satya Shah \\ Applied Engineering and Management \\ Faculty of Engineering \& Science, University of Greenwich \\ Chatham, United Kingdom \\ s.shah@gre.ac.uk
}

\author{
Steffano Mattiuzza \\ Applied Engineering and Management \\ Faculty of Engineering \& Science, University of Greenwich \\ Chatham, United Kingdom \\ s.mattiuzza@gre.ac.uk
}

\begin{abstract}
The paper examines the use of case study analysis of additive manufacturing implementation within small and medium enterprises (SME). Many of the manufacturing enterprises are subjected to lot of disruptive and changing landscape over the years. The current expectations of the new changing markets are shorter product cycles and fulfilling better customization within the produce lines. This allows the entry of Additive Manufacturing (AM) application within many of the product based manufacturing enterprises. AM adopts the process of joining materials through layers to produce objects through $3 \mathrm{D}$ digital data technology that enables it to innovate the traditional manufacturing processes. The paper aims to present an overview of additive manufacturing technology through small and medium enterprise adoption by conducting three mini case studies within manufacturing businesses. The paper aims to investigate how additive manufacturing drives innovation within these manufacturing SMEs and while understanding the adoption of these technological advances, proposes an appropriate implementation framework. The study also aims to evaluate any tactical patterns of innovation and growth towards the adoption and advantages of additive manufacturing, while recommending solutions to address certain challenges, especially within the supply chain transformation within the environment. Finally, through this paper the authors aim to address that use of additive manufacturing technology may or may not be applicable to all manufacturing SMEs.
\end{abstract}

Keywords-Additive Manufacturing (AM); manufacturing SMEs; technological innovation; strategic direction

\section{INTRODUCTION}

Many of the current businesses including that of manufacturing small and medium enterprises are facing a challenging and highly competitive market within global environments. However, it is also seen that many of these small and medium enterprises (SMEs) are also playing a vital role in boosting the local economies through its involvement and constant interaction in society. They are continuously challenged to be more reactive, flexible and globally competitive in the most cost-effective manner [1]. It is also noted that many of these SMEs tend to adopt on the changing market requirements towards shorter time to market products and highly customisable service differentiation within their products. Hence, in the given environments many of these manufacturing SMEs relies upon the use of AM technologies as the most disruptive to address these challenges within any traditional manufacturing or product-based enterprises.

The paper aims to focus their studies with evaluating and understanding the concept of additive manufacturing that can address and provide better solutions to these problems and that of the changing market demands. Many of the current research directions within this subject tends to provide the subject more towards the use of the technology and its implications within the manufacturing sector, however they lack the key direction through subjective, tactical and most importantly the managerial direction required by these manufacturing SMEs. This paper attempts to address this gap through the interaction of first evaluating the understanding of additive manufacturing and then its focussed relation to manufacturing SMEs in the context of case studies to propose a better managerial strategy towards AM adoption.

The first part of the paper examines current and existing literature providing the authors a thorough understanding of additive manufacturing, its current applications and benefits and the key drawbacks towards its adoption. Secondly, the authors also aim to analyse the context of manufacturing SMEs and the key manufacturing strategies. Finally, the authors propose a research framework and methodology with the use of three mini case studies to examine the existing structure of these manufacturing SMEs and to enable the researchers to propose the adoption and implementation of additive manufacturing within their environments. The analysis and findings from these case study will further determine the development of an appropriate method and the framework to support the better implementation and use of additive manufacturing technologies within manufacturing SMEs. With the use of the proposed framework, production and manufacturing managers will be able to utilise and plan the cost and investment strategies within the company more effectively.

\section{LITERATURE REVIEW}

The key focus of this literature study is to briefly examine the foundations of additive manufacturing allowing the researchers to further develop their understanding of these technologies and application and towards its successful adoption and facilitation within global manufacturing environments, especially that of small and medium enterprises. The paper presents the general foundations of their research and through the understanding developed by the literature, it aims to propose a novel framework for SMEs towards a more global and shared network for additive manufacturing technologies to be adopted within these enterprises to allow them to achieve a thorough strategic fit for their globally competitive markets. 


\section{A. Principles of Additive Manufacturing}

The most common definition of AM is presented well by the ASTM Standard which refers to the "process of joining materials to make objects from 3D model data on layer by layer basis compared to subtractive manufacturing methodologies" [2]. Similarly, the concept of rapid prototyping is generally referred to the application of this technology. As the early application of AM it mainly provided faster assistance towards the increase time to market proposition and that towards incorporating innovation within the products. It can also be referred as the process of creating models and prototypes of parts or finished products quicker than traditional manufacturing process [3].

Similar research studies identified that many enterprises are accepting the adoption of additive manufacturing techniques to boost their performance and that of cost saving measures within their environments. The term additive manufacturing also is referred many times as additive processes, additive techniques, layered manufacturing and free-style fabrication in industrial environments $[4,5]$. The concept of AM evolved over the years in different sub categories; they are classified as rapid Manufacturing (RM) evolving from Rapid Prototyping (RP) technologies [6] that were successfully used to physically visualize designs and concepts [7]. Rapid Tooling (RT) combines rapid prototyping techniques with conventional tooling practices with the aim of producing a mould or parts of a functional model quickly time and at a lower cost in comparison with traditional machining methods [6]. At the same time, the freedom of design, new materials and better structural performance allow eliminating totally tools [8]. Research studies focusses towards the development of many different additive manufacturing techniques over the past decade. For example, research studies classified them on the basis of the process of material supplied - liquid, solid or powder-based systems [9]. These broad range of technologies provides many advanced level functionalities and opportunities to manufacturing companies in order to upgrade their portfolio and be able to expand upon their production processes. In the conventional manufacturing environment, Stereolithography (SL), selective laser sinterising (SLS) and selective laser melting (SLM) play the most significant role. Though the high cost of the machinery and still the limited number of suitable materials many expectations come from metal AM techniques that have the potential to disrupt the market, as an alternative or supplementary method of manufacturing. AM finds applications in many sectors and market segments. The following pie chart shows how the revenues for AM are distributed across the market. A large share (consumer products, industrial machines, motor vehicles) interests small and medium manufacturers. Also, the medical and aerospace sectors can be related to advanced niche SMEs and not only to Multinational Companies and Universities. In particular, metal 3D production systems are widely used in these markets.
As per other similar research studies that identified the concept of 3D printing as additive manufacturing has been around for number of years, but more recently been adopted across different business environments due to the advancement in technology [10]. However, as the technology gets more established and widely adopted across different environments and sectors with its vast applications it will eventually have a full acceptable maturity across different sectors. This will further allow the development and implementation of new technological advancements across different markets and sectors likewise [11]. However, the full industrial adoption of additive manufacturing practices also focusses towards many other aspects related to the businesses including that of social, environmental and technical foundations within the sector [12]. It has also been noticed that additive manufacturing also identified the key ethical issues concerning the negative use of the technology towards other printing examples for non-commercial activities. However, AM demonstrates the key advantages towards enhancement and retaining of the skills and knowledge required to improve the economic and social aspects in certain areas. Research studies suggests that AM is useful towards any regional transformation processes and that of the areas of intellectual property with the focus of skills within the companies [13]. The tool allows to modify any existing supply chain by creating new VSs and greater opportunities for enterprises which were not possible earlier with its ability to allow business transformations such as the production and performance analysis [12].

It has widely been noticed that the perception of additive manufacturing is often towards a disruptive technology that aims to change the traditional supply chain processes and structures. Hence, many of the earlier research studies have not been able to fully adapt and implement the technology, while focussing on towards partial adaption in existing manufacturing processes and on "need to use" basis. Similarly, studies have widely been accepted and used for academic purposes towards research studies, educational purposes and that of mechanical/manufacturing demonstration purposes. The full scale adoption and implementation of additive manufacturing technology from commercial usage by replacing traditional methods has yet to be seen. Similarly, our research finds the lack of studies within research community that identified the use of additive technology within other manufacturing processes or that of wider supply chain context. For example, research discussed on various market structures that may be affected through the use of additive manufacturing applications [10]. They further proposed and distinguished monopolistic markets to that of competitive markets and hence suggesting that additive manufacturing will aim to increase profits towards monopolistic markets and conventional manufacturing processes will be more ideal towards the competitive market environments [14]. Other researchers have developed scenarios where AM complements traditional mass manufacturing processes and suggested that mass production benefits from AM where smaller quantities must be isolated from scalable mass productions [15]. Finally, use of new 
business structures through combination of AM with SCM through the direct combination of conventional structures with digital manufacturing practices has also been examined [16].

\section{B. Technology Innovation and Manufacturing SMES}

The use and adoption of additive manufacturing within any aspects of product portfolio management enables different levels of changes within the structure. For example, the redesigning of the business firm especially that of SMEs could be identified as a major form of transformation within the environment with the adoption of additive manufacturing practices. This transformation could be identified as source of innovation within the environment. Studies define innovation as "multi-stage process whereby organisations are able to transform ideas into new and improved products or services, including processes to allow them to advance, compete and differentiate them within the market place [17]. Within an engineering dimension, these processes can start with key focus towards the technical potentials of a new product or a specific tool. SMEs need to adapt and improve their capabilities towards new innovative technologies in order to satisfy the increasing demand and changing requirements towards customisation, responsiveness and quality in high value innovative product market strategies [18]. Research has suggested that there is far more emphasis towards technological innovation as many of the SMEs are businesses are relying on this single aspect of technology and often have been ignoring other approaches to innovation management [19].

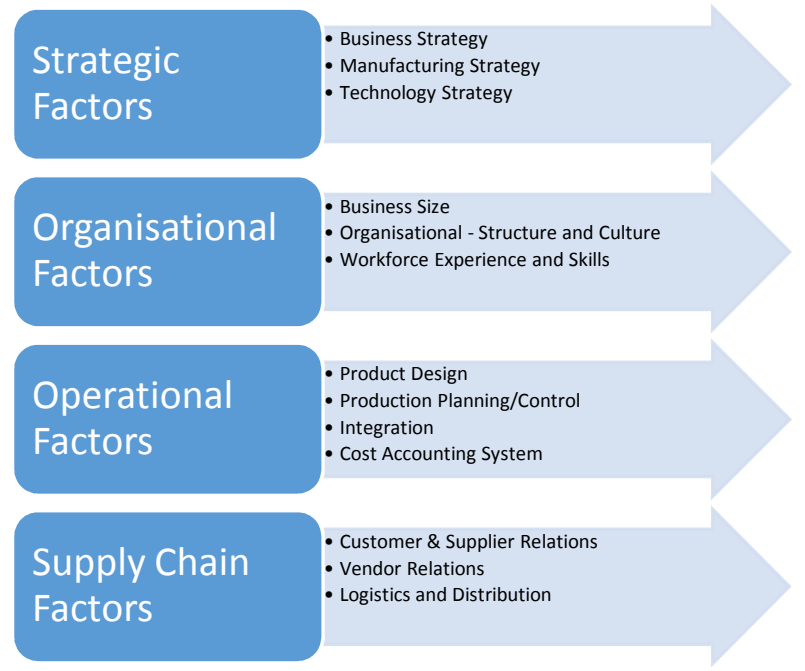

Fig 1. Key Factors for Technology and Innovation in Global Manufacturing

Therefore, the authors of this research aim to propose an innovation paradigm which systematically integrates people and process based issues within the technological innovation in the context of SMEs. This enables cross-functional working environments where multiple roles are shared across different departments. It has also been evident that the practices adopted within larger organisations due to their structure and operations, similar approaches of innovation are not really applicable because many of these innovative practices needs to be contextualised within the environment.

It has also been identified that lack of financial resources and support is one of the key drawbacks towards investing in technological innovation and its adoption. Some of the other drawbacks identified within our study were the lack of governmental policies, credit ratings, training and skills, market awareness and knowledge, skilled and experienced technical people [1]. In order for SMEs to enable any form of continuous improvement and investments within research and development areas is limited due to lack of resources and capabilities [20]. Similarly, research noted that key barriers of lack of adequate resources, and the churning risks associated towards any adoption and implementation of the technology within the SMEs. Many of senior management are more focussed towards the business-as-usual activities rather than exploring the opportunities and benefits of these technological advancements. Similarly, many of these SMEs due to their flexible and simple structure of their operations and product development should be capable to take strategic and tactical decisions to facilitate better customer relationships within the markets. Hence, it can be said that the key strategic advantage for any SME is towards their ability and agility to address the rapidly changing demands and feasibility of the industrial advancements within their environments. It has been seen that technology innovation is considered as an indispensable competitive tool that needs to be integrated through limited resources including the drawback of skills workforce and that of the complex organisational structures. Finally, this research study has identified a fundamental gap in research towards the importance and relevance of additive manufacturing technology is able to respond and address these drawbacks and that of the needs of the company. So it would be true to reflect that the critical points of implementation and growth areas are also important areas for this study.

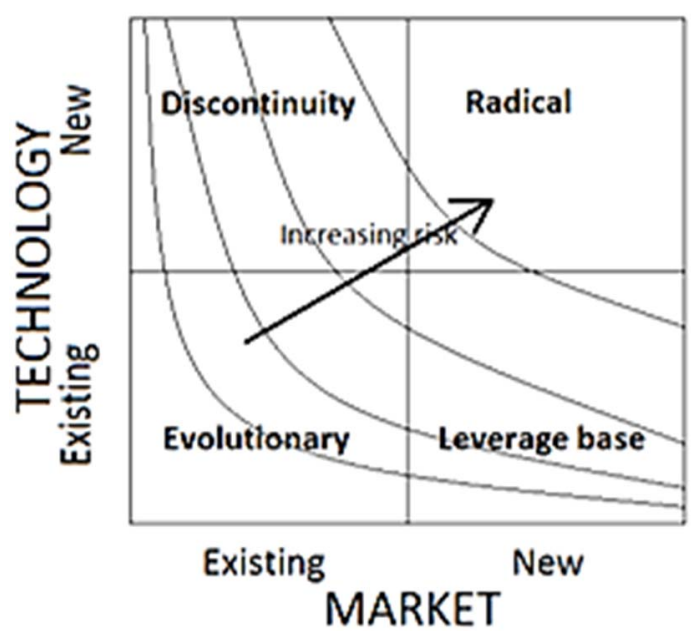

Fig 1. Technology-Market paradigm and risk [21]. 
For example, an important strategic tool is that of the traditional Ansoff matrix tool which provides four growth and innovation paths for any business. This mainly focusses towards existing and any new products, both within existing and new markets. Hence, the resulting strategies from the method derived are: market penetration (existing productexisting market), market development (existing product-new market), product development (new product-existing market), and finally diversification (new product and new market). This concept is briefly adopted by researchers as an attempt to capture technological transformation through the substitution of technology vs. products as shown in figure 2 [21]. It has already been proven that when businesses adopt any radical measures compared to more evolutionary practices the overall risk are higher. Similarly, discontinuity often is represented through new components that are substituted for existing and current technological tools or to enable an increase in productivity and quality with minimal changes to the product functionalities. Through introduction of any existing technologies within new markets enables businesses to minimise or leverage any existing strategies and that of their capabilities for global market expansions. However, in this scenario of new technologies in new markets allows businesses to facilitate wider opportunities and should consider any risks associated with the process. As discussed earlier within our research that additive manufacturing is an existing technology and one which rapidly evolving within different markets through its application and adoption. Innovation and operations strategy of the business should consider all stages of product and market developments, including that of its growth, mature and phasing out aspects carefully [22].

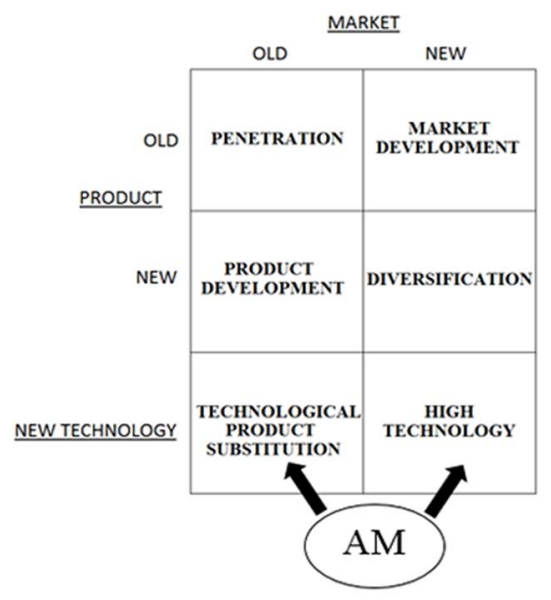

Fig 2. Role of AM in the $3 \times 3$ Ansoff Matrix

Hence, our study aims to present the use of additive manufacturing that enables any potential growth paths through the illustration of the nine cell Ansoff matrix method. The technology feature has been included as a further driver to allow the addition of new dimension to the overall business which is very different to that of the new product or new market dimension. With the introduction of new technologies such as that of additive manufacturing, similarly the knowledge, experience, capabilities, drawbacks and challenges are also going to be new; hence allowing the innovation shift to address the new challenges and that of its varied opportunities. The paper aims to further evaluate new and original paths of innovation which many of the SMEs should aim to adopt towards AM implementation [24].

\section{RESEARCH FRAMEWORK}

This study relies on multiple sources which allowed to pursue a solid method of investigation. It is based on a critical analysis of the previous and current literature and on a multiple case studies approach. By reviewing the most relevant authors and experts in this field, it has been possible to sum up the main aspects regarding the AM in the manufacturing SMEs' context. Moreover, some findings stated by the authors could be challenged. In addition to this, a significant starting point to approach from a managerial perspective of the practice and the implementation of AM in manufacturing SMEs has been conducted [13]. Finally, the case studies have given the biggest contribute to this work by providing the necessary empirical evidence to validate these results and enhance this research.

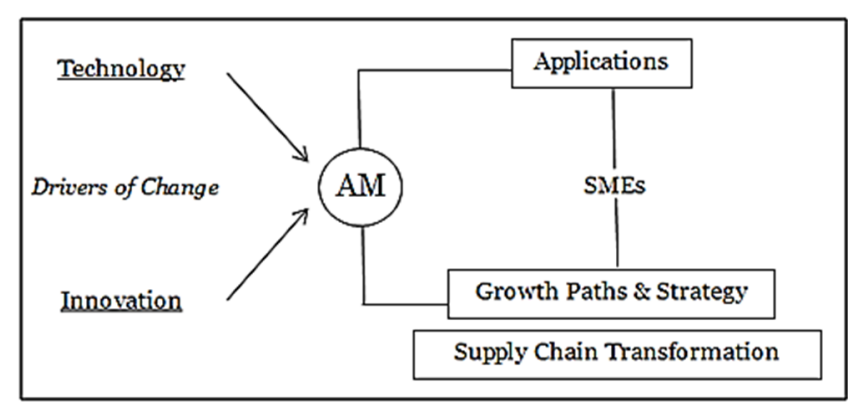

Fig 3. Research Framework for AM Implementation Framework.

The methodology to approach Additive Manufacturing relies first of all on the comprehension of AM, its most upgraded applications and its effective factors for implementation. Then, the understanding of the SMEs scenario, the high contextualization of manufacturing improvements in SMEs. Consequently, the necessity to link supply the chain management to AM and to the strategy of the company. At the base of this process the author individuated two main drivers of change: Technology and Innovation; as shown in the following representation (Figure 3). AM deals with high value-added products and requires new skills and new organisational approaches; therefore, it is not a conventional industrial technology suitable for mass production and wide assembly line. Therefore, to build a model able to understand its entire implementation and support its process planning, it is necessary to combine all the level of the system: from a point of view which includes strategic and supply chain aspects to the operational processes. Indeed, technical aspects are strictly related to managerial and organisational aspects for AM. 


\section{A. Case Study Overview}

Designing the case study methodology is crucial to achieve valid results. The first step is defining whether using a single or a multiple case study structure. A suitable solution for this kind of research is using multiple case studies. First of all, to prevent from the risks of the single case, and also to support the varied exploratory nature of this research [23]. Due to limited availability towards the use of AM in manufacturing SMEs, the authors decided to focus on three cases. In particular, these companies correspond to the type of conventional manufacturing SMEs implementing additive manufacturing (AM) technologies. Furthermore, they were set in two different countries (from UK and Italy), which are both leaders (together with Germany) of this industrial sector in within Europe. The first step to collect information consists of identifying the appropriate source of evidence. For this study the most used are:

- interviews;

- direct observations:

- participant observations;

- technical documents;

- physical artefacts.

The main principle of data collection is "using multiple sources of evidence" [23]. Consequently, the creation of a case study database. Hence, data are rationally analysed. In conclusion, the data collection method is a mixture of a linear-analytic technique and a comparative pattern. Moreover, there is a combination of deductive and inductive approach. Initially, a deductive method was followed to build the first framework of investigation. In a second phase, it was modified and improved by corroborating the empirical evidence provided by the companies. Finally, the author provided managerial outcomes and generalized some findings. In this last stage, it was followed an inductive method. This method is suitable for an explorative and descriptive investigation, structured with a multiple case study design. The literature review allowed the author to gain knowledge about AM and manufacturing SMEs. In specific, regarding papers and studies that describe models to investigate or analyse AM, or even frameworks for its implementation. The following investigation protocol is mostly based upon these previous works.

\section{B. Case Study Analysis}

Once that scope and method have been defined, an intense work of emailing and networking started. At first, the author established research method and targets with the companies. Then, he visited their sites, interviewed and discussed with their CEOs and employees with the aim of:

- Understanding their state of art;

- Developing a better and accurate comprehension of AM to develop and propose IDEF0 model;

- Gaining a complete perspective of issues related to a successful AM implementation and offer some managerial guidelines;

- Investigating an AM product to analyse its advantages and pitfalls.

\section{1) Case Study A - UK Enterprise}

The case study carried out was with a company that specialised as Additive Manufacturing is an independent enterprise which was born as a spin-off of company A Filters Ltd. Although the small dimensions, the rate of innovation and investment in $\mathrm{R} \& \mathrm{D}$ is very elevated. Company $\mathrm{A}$ is a niche business, which provides high value added products in small batches, often less than ten. Moreover, they provide a kind of bespoke solution to their customers. Additive Manufacturing can deliver unique products regarding quality and features.

- The main advantage of AM for Company A is the possibility to produce filters with unique and special features that otherwise could not be realised. The freedom of design is the central point:

- "People want open area and strength in a filter. Often, only AM allows to combine these two characteristics effectively" said the Director.

The Selective Laser Melting 3D Printer (SLM), made in Germany, uses products 316 stainless steel. Company A only uses this kind of powder. They do not believe in cleaning the machine out to make it perfectly ready for another material, because of the risk of contamination.

- Handling Powder: "We are health and safety conscious first of all" (the Director). He was personally involved in workshops to handle in the best way this critical aspect of AM technology. The percentage of un-used powder in some cases is around $80 \%$. Considering that each cycle can cost around $\$ 7000$, the re-use of the powder is a contentious issue. Moreover, the recycling of metallic powder is an attractive feature of AM. For some critical aerospace components, large scale aerospace companies are demanding that powder is only used once and that personal protective equipment, such as full face masks are used once, even if they are made to be re used. This type of rule introduces an unnecessary and exorbitant cost to SMEs. Re-using powders, and masks, may reduce costs and environmental impact. These delicate internal operations need monitoring and control.

- Maintenance: the machine requires very little maintenance. Common and simple problems are fixed internally within the company. Problems are related to the laser or the scanner system, the company relies totally on the supplier. Usually it needs to be sent back to the manufacturer. This phase takes even weeks stopping the machine work. Having the supplier geographically close can be helpful.

- Modifications and settings: having an Open machine is essential to be flexible also during the R\&D phases. According to Company A, except for a short initial training, they did not get much support from the 
supplier surprisingly regarding machine settings and design.

- The role of Design: most of the defectives are due to a poor design rather than a machine failure. This is the fundamental skill barrier. The main complications regard the support structure and the orientation of the cellular structures. Much experience is gained through R\&D programs. However, advanced and specialist software requires significant investments. Also, software maintenance costs are ongoing every year and are expensive.

- Post-Processing: these operations become central when there are big batches and mass production. Having been a conventional manufacturer for years, Company A has in-house several machines and tools to do these operations. Moreover, they have a simple and effective polishing machine built internally.

\section{2) Case Study B - Italian Enterprise}

Company $\mathrm{B}$ is an Italian manufacturing SME, which works in the mechanical sector since 1975 . Nowadays, there are 43 employees working in a plant of $8800 \mathrm{~m} 2$. In 2015, the company enlarged their wide production portfolio by purchasing a 3D Metal Printer and later a 3D Plastic Printer. Company B operates in the mechanical sector to deliver highvalue added products and high-quality solutions. In particular, they build special types of machinery, machine components and tools, process automation.

Company B saw in the new capabilities enabled by the Additive Manufacturing an excellent opportunity to expand their skills and to expand to new markets and sectors in which they are not present; in fact, in the segments where they are currently working, this technology is not intensely demanded and useful. Indeed, upgraded conventional technologies (CNC, removal machinery) are efficient and adequate. They are looking at new markets: aerospace, medical, biomedical, and every sector which needs additive solutions. The purchasing of the Plastic 3D printer at a second step testifies the willingness to cover this segment broadly, to meet a large demand and to diversify.

- The main advantage: the new technological capabilities and consequently the possibility to approach new markets;

- The main barriers: the initial investment and the weak knowledge about this disruptive and fresh technology.

The Laser Melting System Machine applies metal powder bed fusion technology [2]. It uses stainless steel. Build volume of $250 \mathrm{~mm} \times 250 \mathrm{~mm} \times 300 \mathrm{~mm}$. The plastic 3D printers are one of the most advanced for production regarding quality, precision and structural characteristics (SLS system, build chamber: 298 x 185 x 200mm).

- Handling Powder: currently, they are purchasing the powder from the machine supplier. Before purchasing the machine, they were totally aware of the criticality of this material. Indeed, they planned to set up the machine in a specific area of the site, and to build a special room for the powders. It is insulated, with an own dedicated ventilation system, ready to be modified according to the ATEX rules in case they would start to use aluminium or titanium powders, for instance. This kind of materials can be managed since the company estimates and make plans regarding how to handle them in advance.

- Maintenance and Modifications: In the case of problems related to the laser or the software, the provider has always been available and responsive. According to Company $\mathrm{B}$, the $3 \mathrm{D}$ plastic machine is more "closed".

- Design and pre-processing: The role of design is crucial. The structural design is the first step to obtain a good product: shape, orientation, supports are the most critical aspects of printing the product. Time to learn: Before going to the market, Company B spent several months to learn how to design effectively $3 \mathrm{D}$ parts; that was the biggest effort. Now they have gained the adequate knowledge to advise how to improve the product and reduce the printing costs, by modifying the design. These pre-processing operations are necessary to be competitive.

- Software: Software upgrade costs are relevant. The company is waiting to understand what kind of customers and products will become strategic for them, in order to acquire more advanced and specific software, consequently more expensive and difficult to use.

- Post-Processing: these operations take time; however, this is the strong point of Company $\mathrm{B}$, which has inhouse the technologies and the capabilities to do efficiently and accurately all the finishes and the corrections (i.e. grinding, quenching, and surface finishing), to analyse in detail the properties of the material, to add further mechanic operations. This strategic condition allows the Company to provide responsively finished products to the customers and to build even end-use objects. These considerations played a fundamental role when they decided to invest in this technology.

\section{3) Case Study C-Italian Micro Enterprise}

The case study $\mathrm{C}$ is a small injection moulding company founded in 1993 based in Turin (Italy), in an area of about 1,200 square meters. The company is divided in two main departments: a mechanic's workshop equipped with several $\mathrm{CNC}$ machines, and a production area where there are 20 injection moulding machines. In 2016, case C adopted a plastic 3D Printer, to enhance the product design and development. In the context of this research, the adoption of 
a plastic $3 \mathrm{D}$ printer is less relevant compared to a metal 3D machine; it is just a first step towards this kind of innovation. However, it arose the occasion to further implement this technology by producing a mould through AM. Hence, the opportunity to carry out a cost analysis for this company came out, with the purpose of estimating the advantage of this operation. For the highlighted reasons, this case is examined from a different perspective, more technical and less strategic with the aim of:

- Showing a different environment and way to implement the Additive technology.

- Analysing the costs of a product made through AM to provide practical evidence of its advantages and drawbacks.

The most relevant aspect to highlight is the lack of precision that occurs sometimes. Indeed, for small technical parts that require high level of detail the machine can be unsatisfying. To avoid this problem, it is suggested to increase the infill layer density. However, this setting implicates a considerable growth of production time. This is a limit, since a few grams component requires up to 5-6 hours.

- Maintenance: The machine requires very little maintenance. It is very simple to fix and modify except for the internal software.

- Design: design is crucial to achieve good results. In particular, the difference is made by the use of an advanced software that allows to design supports and slicing operations properly.

- Post-processing: the external quality of the printed part is usually very low. It requires some post processing operations; in particular, removing the supports and refinishing the surface.

- Supply Chain: Having this capability internally, shortens the product design and reduces costs, because the company does not need to outsource prototypes.

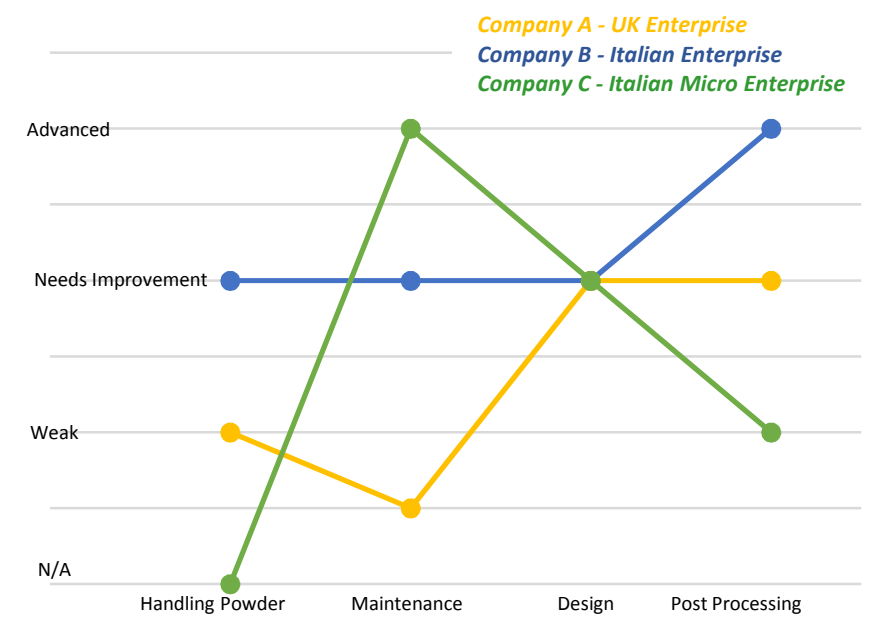

Fig 4. Comparison of the three Case Companies Regarding AM Features
The comparison of the three highlighted case studies is illustrated in figure (4) and as it is obvious, Company A requires more effort regarding re-usage and recycling of the handling powders. Company $\mathrm{B}$ seems to be in a more stable situation regarding adoption of general aspects of the AM features, while Company $\mathrm{C}$ has got a reasonable performance regarding maintenance and modifications.

The above case study discussions present an interesting aspect towards the adoption and use of additive manufacturing and the implementation barriers for many of the manufacturing SMEs. While discussions with the management and its employees, once identified the real advantage that AM can deliver, three main problems with its implementation were recognised, including cost of entry; educating the customers: and gaining the essential skills regarding design and machine handling. Those can be achieved through new assets, open innovation, and lots of experience and R\&D. Similarly, within the case study (b), the company was able to face the organizational change in terms of HR, layout, logistics thanks to the ability of the management in finding out and consequently planning and forecasting the transformation. Moreover, although company (b) remains a typical SME, it is very well structured and organized because the high the rate of innovation led them to become accurate on these aspects. Given their current skills and environment, they can support the AM production with the post-processing operations, the service and the integration with conventional manufacturing system in which they are experts. Therefore, company (b) identified that this technology could become strategic in the near future and pave the way for new markets and new businesses opportunities. AM will bring diversification and innovation. And finally, company (c) was strongly interested in AM and the benefits that it can deliver to the plastic producers. In particular, in the injection moulding sector, the most interesting feature was the possibility to build complex cooling systems inside mould' $s$ parts, in order to reduce the cycle time and also enhance the quality of each product. Consequently, to save money, reduce costs and becoming more competitive.

\section{CONCLUSION}

The paper aims to cover an important aspect on the overview of additive manufacturing techniques and that of the key implications towards the implementation of the technology towards more traditional and conventional manufacturing SMEs. Some of the key advantages and its drawbacks, and opportunities and barriers towards its implementations are explored within the paper. The key purpose of this study was to conduct a comprehensive review of additive manufacturing through the means of an industrial approach, with specific focus towards manufacturing SMEs. It also covered some of the more fundamental references and key starting points towards the adoption and implementation of the technology. Secondly, it was also possible to categorize growth and innovative tactical paths that were accepted through the use of additive manufacturing. These are further connected to the technological innovation and that of the 
transformation measures for supply chain that are aimed towards generation or exploitation of the technology advancements. There is also a requirement to better understand the actual and suitable possibilities, practices and wider opportunities towards more conventional manufacturing SMEs to adopt and integrate additive manufacturing technologies. Future studies should target more practical case studies to facilitate a thorough understanding of AM adoption and integration by SMEs. Similarly, it is also important to evaluate new and original paths of innovation that enterprises are adopting towards the implementation of additive manufacturing applications. However, there is still lack of research evidence towards the strategic implementation of supply chain management practices in context of additive manufacturing. The balance between organizational and technological factors, the factors affecting supply chain and in relation to AM suppliers and customer relationships and that of the management and supply of supplier relationships has not been fully covered through existing research studies. As the rapidly evolution and customization of the additive manufacturing technology requires proper control measures with respect to its full adoption, the further evolution and innovative applications of the technology are yet to be fully explored within SMEs to provide better customer experience in the market place.

Acknowledgements This paper is part of an ongoing research project within the research centre and includes a case study research on the adoption and implementation of additive manufacturing (AM) technology in manufacturing SMEs. The authors aim to propose a framework that will assist as a tool for manufacturing managers towards better understanding and utilisation of resources of technology based projects including the use of additive manufacturing technology within their environments.

\section{REFERENCES}

[1] J.Y. Farsi, and M.T. Toghraee, "Identification the main challenges of small and medium sized enterprises in exploiting of innovative opportunities (Case study: Iran SMEs)," Journal of Global Entrepreneurship Research, 2(1), pp.1-15, 2014.

[2] ASTM Standard, ISO / ASTM52900-15, Standard Terminology for Additive Manufacturing - General Principles - Terminology, ASTM International, West Conshohocken, PA, 2015.

[3] M. Attaran, "The rise of 3-D printing: The advantages of additive manufacturing over traditional manufacturing," Business Horizons, 60(5), pp.677-688, 2017.

[4] L. Beltrametti, and A. Gasparre, La stampa 3D come nuova sfida tecnologica al lavoro manifatturiero. 26 Convegno annuale di Sinergie. 2014.

[5] B. Berman, "3-D printing: The new industrial revolution," Business horizons, 55(2), pp.155-162, 2012

[6] C. Achillas, D. Aidonis, E. Iakovou, M. Thymianidis, and D. Tzetzis, "A methodological framework for the inclusion of modern additive manufacturing into the production portfolio of a focused factory," Journal of Manufacturing Systems, 37, pp.328-339, 2015.

[7] C. Tuck, R. Hague, and N. Burns, "Rapid manufacturing: impact on supply chain methodologies and practice," International Journal of Services and Operations Management, 3(1), pp.1-22, 2006.
[8] C. Weller, R. Kleer, and F.T. Piller, "Economic implications of 3D printing: Market structure models in light of additive manufacturing revisited," International Journal of Production Economics, 164, pp.43$56,2015$.

[9] K.V. Wong, and A. "Hernandez, review of additive manufacturing," ISRN Mechanical Engineering, 2012.

[10] P. Merrill, “The new revolution," Quality Progress, 47(1), pp.50. 2014.

[11] J. Beaman, D. Bourell, and D. Wallace, "Additive manufacturing (AM) and 3D printing," Journal of Manufacturing Science and Engineering, 136(6), pp.060301, 2014.

[12] B. Rylands, T. Böhme, III, R. Gorkin, J. Fan, and T. Birtchnell, "The adoption process and impact of additive manufacturing on manufacturing systems," Journal of Manufacturing Technology Management, 27(7), pp.969-989, 2016.

[13] P. Li, S. Mellor, J. Griffin, C. Waelde, L. Hao, and R. Everson, "Intellectual property and 3D printing: a case study on 3D chocolate printing," Journal of Intellectual Property Law and Practice, 9(4), pp.322-332, 2014.

[14] C.F Durach, S. Kurpjuweit, and S.M. Wagner, "The impact of additive manufacturing on supply chains," International Journal of Physical Distribution and Logistics Management, 47(10), pp.954-971, 2017.

[15] A. Sasson, and J.C. Johnson, "The 3D printing order: variability, supercenters and supply chain reconfigurations," International Journal of Physical Distribution and Logistics Management, 46(1), pp.82-94, 2016.

[16] J. Holmström, and J. Partanen, "Digital manufacturing-driven transformations of service supply chains for complex products," Supply Chain Management: An International Journal, 19(4), pp.421430, 2014.

[17] A. Baregheh, J. Rowley, and S. Sambrook, "Towards a multidisciplinary definition of innovation," Management Decision, 47(8), pp.1323-1339, 2009.

[18] R. Ali, and A. Mustafa, A Strategy to deploy rapid prototyping within SMEs (Doctoral dissertation, Bournemouth University), 2013.

[19] R. McAdam, W. Keogh, R.S. Reid, and N. Mitchell, "Implementing innovation management in manufacturing SMEs: a longitudinal study," Journal of Small Business and Enterprise Development, 14(3), 385-403, 2007.

[20] A.M. Romouzy-Ali, S. Noroozi, P. Sewell, and T. Humphries-Smith, "Adopting rapid prototyping technology within small and mediumsized enterprises: The differences between reality and expectation," International Journal of Innovation, Management and Technology, 3(4), pp.427, 2012.

[21] W. Boulton, and A. Nair, Changing Product-Market Paradigms Implications for Operations Strategy, 2006.

[22] A. Nair, and W. R. Boulton, "Innovation-oriented operations strategy typology and stage-based model," International Journal of Operations and Production Management, 28(8), pp.748-771, 2008.

[23] R.K. Yin, Applications of case study research. Applied social research methods series. Thousand Oaks: Sage Publications, 2003.

[24] S.Shah, S. Mattiuzza, E. Naghi Ganji, and Coutroubis, A, "Contribution of additive manufacturing systems to supply chain", In Industrial Engineering, Management Science and Application (ICIMSA), 2017 International Conference on IEEE, pp.1-5 